\title{
Organization of near-inertial energy by an eddy field
}

\author{
By PATRICE KLEIN ${ }^{1 *}$, STEFAN LLEWELLYN SMITH ${ }^{2}$ and GUILLAUME LAPEYRE ${ }^{1}$ \\ ${ }^{1}$ Laboratoire de Physique des Océans, IFREMER, France \\ ${ }^{2}$ Department of Mechanical and Aerospace Engineering, UCSD, La Jolla, USA
}

(Received 23 December 2002; revised 10 September 2003)

\section{SUMMARY}

We propose an analytical solution for the evolution of the spatial variability of wind-forced inertial energy in the presence of an oceanic mesoscale eddy field. The solution requires knowledge of only the stream function of the eddy field and the Rossby radii of the normal modes associated with the near-inertial oscillations. It also shows that, when the vorticity spectrum slope is shallower than $k^{-4}$, inertial energy is trapped inside structures in which the Laplacian of the vorticity field is positive and whose size matches a critical length-scale that increases with time. Numerical simulations using different models, including a fully nonlinear shallow-water model, confirm the analytical results.

KEYWORDS: Inertial oscillations Mesoscale eddies Spatial organization

\section{INTRODUCTION}

Near-inertial oscillations (NIOs) in the upper oceanic layers are mostly forced by intermittent atmospheric storms whose scales are of the order of $1000 \mathrm{~km}$. However, since Weller (1982), it has been recognized that the presence of mesoscale eddies can distort the NIOs and decrease their length-scales down to values smaller than $100 \mathrm{~km}$ within a few days. Numerous subsequent studies devoted to this NIO distortion have stressed the important role of the relative vorticity associated with the eddies, and explained how the NIOs are expelled from cyclonic structures and trapped within anticyclonic ones (Kunze 1985; Balmforth et al. 1998; Van Meurs 1998). Most of these studies have considered isolated eddies characterized by only one specific lengthscale. In this case, the interactions between NIOs and eddies are straightforward to study and a Wentzel-Kramers-Brillouin (WKB) analysis is sufficient to represent them. Following Young and Ben Jelloul (1997) (hereafter YBJ97), Klein and Llewellyn Smith (2001) (hereafter KLS01) used a different approach that considers a large number of strongly interacting eddies. Such a turbulent eddy field is characterized by a continuous wave-number spectrum, i.e. one with energetic length-scales ranging from 200 to $10 \mathrm{~km}$. KLS01 showed that it is not just one length-scale, but a large range of scales related to the turbulent eddy field that is involved in the NIO distortion. Hence the dynamics of the NIOs are more complex than those displayed by previous studies. Both approaches (isolated eddies or turbulent eddy field) are relevant to ocean dynamics. The latter is however becoming increasingly more pertinent. Many recent studies have shown the existence in the oceans of a large number of strongly interacting eddies, not only in well-known 'eddy regions' such as the Gulf Stream or the Antarctic Circumpolar Current, but also in a large number of other regions as revealed by recent satellite and in situ data (Stammer 1997; Wunsch 1997; Rudnick 2001) and very high resolution $\left(1 / 64^{\circ}\right)$ numerical simulations (Hurlburt and Hogan 2000; Siegel et al. 2001). One important property of such eddy fields, as given by the classical arguments of geophysical turbulence (Hua and Haidvogel 1986), is that the vorticity spectrum slope is usually close to $k^{-2}$, while isolated eddies have a much steeper spectrum slope (typically $k^{-6}$ ).

\footnotetext{
* Corresponding author: Laboratoire de Physique des Océans, IFREMER, 29280 Plouzané, France. e-mail: pklein@ifremer.fr
}

(c) Royal Meteorological Society, 2004. 
Following the approach of YBJ97 and KLS01, the present paper focuses on the spatial organization of the kinetic energy of the NIOs by a turbulent eddy field. This topic has not been specifically addressed so far. Its importance is emphasized by its strong impact on mixing of the oceanic upper layers. We first summarize what has been found so far on the distortion of NIOs by a turbulent eddy field. YBJ97 proposed a theoretical procedure that, unlike WKB theory, does not assume a priori a horizontal-scale separation between NIOs and the background mesoscale flow. This procedure filters out the inertial period and gives an equation for the slower subinertial evolution of the complex amplitude $A$ defined by

$$
u+\mathrm{i} v=\mathrm{e}^{-\mathrm{i} f t} A,
$$

where $\mathrm{i}^{2}=-1,(u, v)$ is the horizontal velocity of NIOs and $f$ the Coriolis frequency. Using this framework and a fully turbulent eddy field, KLS01 showed that the $A$-spectrum displays a critical length-scale that conspicuously separates the $A$-field into large-scale structures and small-scale structures with very different properties. From a decomposition of the NIO field into

$$
A=\mathcal{R} \mathrm{e}^{\mathrm{i} \theta},
$$

with $\theta$ the phase and $\mathcal{R}$ the amplitude, further numerical and theoretical analysis revealed that the large-scale $A$-structures mostly result from the effects of the eddy vorticity on the phase, whereas their amplitude is unchanged. On the other hand, the small-scale $A$-structures, mostly determined by horizontal dispersive effects, are close to the stream function field at these scales, a result similar to one found in YBJ97. The total $A$-variance was found essentially to be dominated by the large-scale part. However no investigation was undertaken in KLS01 to elucidate whether the spatial structure of the inertial energy, i.e. $\mathcal{R}^{2}$, resembles the vorticity field (as for the large-scale part of $A$ ) or the stream function field (as suggested by YBJ97). The present paper addresses this important question in the following form: what is the spatial structure of inertial energy when a turbulent eddy field is present?

In section 2, we develop an analytical solution for the spatial structure of the inertial energy. Its robustness is checked in section 3 with numerical results using the quasigeostrophic (QG) model of Hua and Haidvogel (1986). We then compare in section 4 this analytic solution with a shallow-water model that uses the primitive equations and is therefore free from the YBJ97 assumptions. A discussion is offered in section 5 and conclusions are outlined in section 6 .

\section{FORMULATION OF THE NIO DISPERSION PROBLEM}

\section{(a) NIO dynamics}

We assume that the mesoscale eddy field is barotropic and QG with a Rossby number, $\epsilon \ll 1$. One consequence is that any dynamical quantity (velocity, vorticity, etc.) related to the eddy field can be expressed directly in terms of its stream function, $\phi$. The horizontal length-scale of the NIOs, $L$, is the same as that of the eddy field, and the NIOs are assumed to be captured by a single baroclinic mode whose Rossby radius is $r_{\mathrm{d}}$, with $\left(r_{\mathrm{d}} / L\right)^{2}=\mathcal{O}(\epsilon)$. These scalings allow us to use the simplest equation for the NIOs derived by YBJ97 in the presence of a background QG flow:

$$
\frac{\partial}{\partial t} A+\mathrm{J}(\phi, A)-\frac{\mathrm{i} h}{2} \nabla^{2} A+\frac{\mathrm{i} Z}{2} A=0,
$$


where $h=f r_{\mathrm{d}}^{2}, \nabla$ is the two-dimensional gradient operator and $\mathrm{J}$ the Jacobian operator, $\mathrm{J}(a, b) \equiv a_{x} b_{y}-a_{y} b_{x}$. The second term in (2) corresponds to advection by the QG flow, the third to dispersion and the fourth to refraction by the QG vorticity, $Z \equiv \nabla^{2} \phi$. We ignore the beta-effect in (2), since it is not important on the time-scale of a few days that we consider here.

Since we focus on the dispersion of the NIO kinetic energy, we again write $A=\mathcal{R} \mathrm{e}^{\mathrm{i} \theta}$. The kinetic energy, $u^{2}+v^{2}=\mathcal{R}^{2}$, and the phase, $\phi$, evolutions are obtained from the following two coupled equations (deduced from (2)):

$$
\left.\begin{array}{c}
\frac{\partial \mathcal{R}}{\partial t}+\mathrm{J}(\phi, \mathcal{R})=-\frac{h}{2}\left\{2 \nabla \mathcal{R} \cdot \nabla \theta+\mathcal{R} \nabla^{2} \theta\right\} \\
\frac{\partial \theta}{\partial t}+\mathrm{J}(\phi, \theta)=-\frac{Z}{2}+\frac{h}{2}\left\{\frac{\nabla^{2} \mathcal{R}}{\mathcal{R}}-(\nabla \theta)^{2}\right\} \cdot
\end{array}\right\}
$$

One important property of (2) is that the integral of the NIO kinetic energy ( $A A^{*}$ or $\mathcal{R}^{2}$ ) over the spatial domain is conserved (Metzger 1999). This can be seen directly by multiplying (2) and its conjugate by $A^{*}$ and $A$ respectively and integrating their sum over the spatial domain. This conservation property implies that the eddies redistribute spatially the initial NIO kinetic energy.

In the following analysis, we consider uniform initial conditions for the kinetic energy $\left(\mathcal{R}_{0}^{2}=1\right)$, which is therefore the steady mean value of the kinetic energy (since the integrated kinetic energy is conserved). Consequently the subsequent analysis focuses on the time evolution of the spatial heterogeneity of $\mathcal{R}^{\prime}$, the departure of $\mathcal{R}$ from $\mathcal{R}_{0}$ :

$$
\mathcal{R}^{\prime}=\mathcal{R}-\mathcal{R}_{0} .
$$

\section{(b) Analytical solution for the NIO kinetic energy}

As in KLS01, we focus on the evolution of the NIO field on the time-scale $t_{s} \equiv \mathcal{O}\left(\{\epsilon f\}^{-1}\right)$ (which corresponds to a few days in dimensional units), starting from uniform initial conditions for the kinetic energy $\left(\mathcal{R}_{0}^{2}=1\right)$. We assume that, during this time period, the advection by the QG flow is negligible and the departure of the NIO field from the initial condition, $A_{0}$, is small. We write $A=\left(\mathcal{R}_{0}+\mathcal{R}^{\prime}\right) \mathrm{e}^{\mathrm{i}\left(\theta_{0}+\theta\right)}$ with $A_{0}=\mathcal{R}_{0} \mathrm{e}^{\mathrm{i} \theta_{0}}$ the uniform initial condition, and assume that $\mathcal{R}^{\prime} \ll \mathcal{R}_{0}$ and $\theta^{\prime} \ll \theta_{0}$. With these approximations, (3) give:

$$
\frac{\partial^{2} \mathcal{R}^{\prime}}{\partial t^{2}}+\frac{h^{2}}{4} \nabla^{4} \mathcal{R}^{\prime}=\frac{h}{4} \mathcal{R}_{0} \nabla^{2} Z
$$

This is the equation of an oscillator with period $\mathcal{O}\left(t_{s}\right)$, forced by the Laplacian of the vorticity field. Hence for very early times $\left(t \ll t_{s}\right)$, the solution for $\mathcal{R}^{\prime}$ should resemble $\nabla^{2} Z$ multiplied by a factor $t^{2}$. On the other hand, an asymptotic limit would be a steady solution where $\mathcal{R}^{\prime}$ resembles the stream function. However, because the eddy field is characterized by a continuous wave-number spectrum, the integration of (4) in physical space is too difficult to treat exactly.

An alternative to get a solution for (4) is to work in Fourier space to obtain a solution $\mathcal{R}^{\prime}$ for each wave number $k$ (i.e. the $\mathcal{R}^{\prime}$-spectrum), and then to come back to physical space. This is possible because in this work, as in KLS01, (4) does not involve any triad interactions and therefore the solutions for the different wave numbers decouple. Using

$$
X(x, y, t)=\sum_{p=-N}^{p=N} \sum_{q=-N}^{q=N} \widehat{X}_{p q}(t) \mathrm{e}^{\mathrm{i}(p x+q y)},
$$


with $X$ any variable, $\widehat{X}$ a Fourier component of $X, p$ and $q$ the horizontal wave numbers and $N$ the total number of horizontal Fourier modes, (4) becomes:

$$
\frac{\mathrm{d}^{2} \widehat{\mathcal{R}}_{k}^{\prime}}{\mathrm{d} t^{2}}+\frac{h^{2}}{4} k^{4} \widehat{\mathcal{R}_{k}^{\prime}}=-\frac{h}{4} \mathcal{R}_{0} k^{2} \widehat{Z_{k}},
$$

with $k^{2}=p^{2}+q^{2}$ and $\widehat{\mathcal{R}_{k}^{\prime}}$ a shorthand for $\widehat{\mathcal{R}_{p q}^{\prime}}$. (5) is the equation of a forced oscillator with period $T_{k}=4 \pi / k^{2} h$. The solution for (5) with vanishing initial condition is:

$$
{\widehat{\mathcal{R}^{\prime}}}_{k}=-\frac{\mathcal{R}_{0} \widehat{Z_{k}}}{h k^{2}}\left(1-\cos \frac{2 \pi t}{T_{k}}\right) .
$$

The solution (6) shows that $\mathcal{R}^{\prime}$ is $180^{\circ}$ out of phase with the vorticity, and consequently the inertial energy is concentrated in negative vorticity regions. The maximum amplitude of $\widehat{\mathcal{R}}_{k}{ }_{k}$ is attained when $t=T_{k} / 2$. The solution (6) allows us to estimate the $\mathcal{R}^{\prime}$-spectrum at a given time $t$. For that purpose we define at time $t$ a critical wave number, $k_{\mathrm{c}}$, (as in KLS01) by

$$
k_{\mathrm{c}}^{2}=\frac{2 \pi}{h t} .
$$

This allows us to partition the spectral space into two regions, the first corresponding to the wave numbers $k \ll k_{\mathrm{c}}$, for which the $\mathcal{R}^{\prime}$-amplitude is still growing in time (because $T_{k} \gg t$ ), and the second corresponding to $k \gg k_{\mathrm{c}}$, for which the $\mathcal{R}^{\prime}$-amplitude has reached a periodic solution (because $T_{k} \ll t$ ). It should be noted that, as in KLS01, we focus on the spectral region corresponding to $k>k_{0}$ (with $k_{0}$ the wave number of the peak of the vorticity spectrum, i.e. the dominant scale of the eddy field) where the vorticity spectrum can be approximated as $\left|\widehat{Z}_{k}\right|^{2} \approx \alpha^{2} k^{-n}$, with $\alpha$ a constant and $n$ assumed to be smaller than 4 . Then, from (6), the simplified solutions of $\left|\widehat{\mathcal{R}}_{k}^{\prime}\right|^{2}$ are

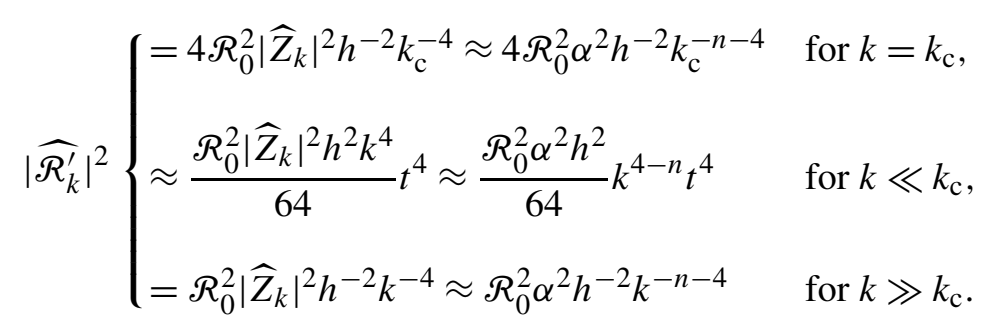

(The third solution experiences oscillations about the mean value shown.) The estimation of the $\mathcal{R}^{\prime}$ spectrum, using (8), reveals a peak at $k=k_{\mathrm{c}}$ when $n<4$. This indicates a scale selection; $\mathscr{R}^{\prime}$ has its largest magnitude in structures whose scales are close to $k_{\mathrm{c}}^{-1}$. For $k \ll k_{\mathrm{c}}$, the $\mathcal{R}^{\prime}$-spectrum has a positive slope and resembles the $\nabla^{2} Z$-spectrum (since $\left|\widehat{\nabla}^{2} Z_{k}\right|^{2}=k^{4}\left|\widehat{Z}_{k}\right|^{2}$ and $\left|\widehat{Z}_{k}\right|^{2} \approx \alpha^{2} k^{-n}$ ). This part of the spectrum increases with time like $t^{4}$. For $k \gg k_{\mathrm{c}}$ the $\mathcal{R}^{\prime}$-spectrum has a steep negative slope and resembles the stream function spectrum (since $\left|\widehat{\phi}_{k}\right|^{2}=k^{-4}\left|\widehat{Z}_{k}\right|^{2}$ ). Since the total NIO kinetic energy is equal to the integration of the $R^{\prime}$-spectrum over the wave-number range, a simple calculation clearly shows that the first part of the spectrum $\left(k<k_{\mathrm{c}}\right)$ is the dominant contribution.

An estimation of the solution in physical space can be obtained from (8). A first estimate can be derived by taking only the most energetic part of the spectrum, corresponding to $k_{0}<k<k_{\mathrm{c}}$. In that case, the analytical expression for the NIO kinetic energy in physical space can be obtained using (7) and (8) integrated in the range 
$k_{0}<k<k_{\mathrm{c}}$. Taking into account the fact that NIOs are mostly trapped in anticyclonic structures (see (6)), we obtain:

$$
\frac{\mathcal{R}^{\prime}}{\mathcal{R}_{0}}=\max \left(\frac{h t^{2}}{8} \nabla^{2} Z_{T},-1\right),
$$

with $\nabla^{2} Z_{T}$ the Laplacian vorticity field truncated so as to retain only the wave numbers $k$ such that $k \leq k_{\mathrm{c}}$, with $k_{\mathrm{c}}$ given by (7). It is important to stress that this analytical solution strongly differs from those proposed by previous studies; it involves the Laplacian of the vorticity and not the vorticity (Kunze 1985), the stream function (YBJ97) or the vorticity gradient (Van Meurs 1998). Furthermore, this analytical solution involves a critical wave number, $k_{\mathrm{c}}$, that separates the large scales $\left(k<k_{\mathrm{c}}\right)$, whose energy still increases in time, from the small scales $\left(k>k_{\mathrm{c}}\right)$, whose contribution is neglected (since from (8) they do not evolve further and are much less energetic). The difference between our analytical solution and previous ones is that ours strongly depends on the fact that our vorticity spectrum slope is gentler than $k^{-4}$. This assumption is quite relevant for a field of strongly interacting eddies since, as mentioned earlier, geophysical turbulence arguments state that the vorticity spectrum slope of such eddy field is close to $k^{-2}$ (Hua and Haidvogel 1986). In the previous studies, this spectrum slope was assumed much steeper since only isolated eddies were considered. We have checked that our analytical solution compares well with the numerical integration of the simplified equation (4), which confirms that the small scales $\left(k>k_{\mathrm{c}}\right)$ have a negligible effect on the solution. The comparison with the full equations (3) is discussed in the next section.

\section{NUMERICAL ASSESSMENT USING THE YBJ97 EQUATION}

\section{(a) Numerical results}

To check the robustness of our analytical solution (9), we have revisited the numerical simulations described in KLS01. Seven-day simulations were carried out using the QG spectral model of Hua and Haidvogel (1986), with (2) incorporated and a horizontal resolution of $256 \times 256$. The QG flow used in (2), assumed homogeneous over the water column, is taken from the three-dimensional QG model at $200 \mathrm{~m}$. The velocity-scale of the QG flow is $U=0.12 \mathrm{~ms}^{-1}$ and $f=0.7 \times 10^{-4} \mathrm{~s}^{-1}$. The length-scale associated with the domain size is $L=350 \mathrm{~km}$. The eddy advective time-scale is of the order of 33.8 days. The QG flow evolves with time but its evolution over 7 days is weak. The resulting QG vorticity field (Fig. 1(a)) is characterized by small-scale coherent vortices and strong vorticity fronts. Its RMS value is close to $0.1 f$. Its spectrum (not shown) has a peak at $k_{0}=5$ ( $k=1$ corresponds to the length of the domain), and a spectral slope close to $k^{-1.7}$ (i.e. much shallower than $k^{-4}$ ) for wave numbers larger than this peak, i.e. for the small-scale part of the spectrum. Just two baroclinic NIO modes are considered in the present study: the third and the fifth with their Rossby radii equal to 16 and $8 \mathrm{~km}$ respectively. The NIO kinetic energy is initially homogeneous over the whole domain with $\mathcal{R}_{0}=1$ for the two NIO modes. Numerical results are shown in nondimensional units (except for the time). During the first few days, the NIO modes' evolution is quite significant since $\mathcal{R}^{\prime}$ attains the order of $\mathcal{R}_{0}$ at $t=3.5$ days. It is important now to examine whether these numerical results confirm the analytical analysis presented in the preceding section.

\section{(b) Comparison with the analytical solution in spectral and physical spaces}

First, the characteristics of the $\mathcal{R}^{\prime}$-spectra deduced from the numerical results (Fig. 1(b)) compare well with the analytical estimates (7)-(8). Wave-number values of 
(a)
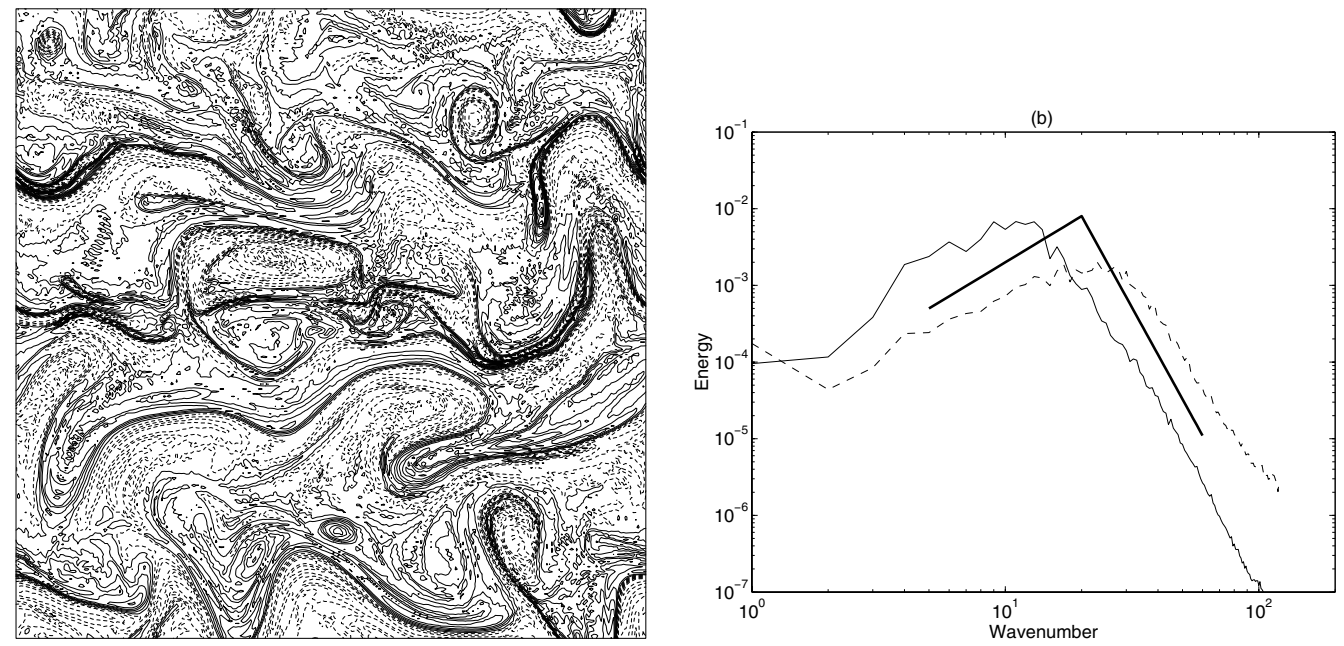

Figure 1. (a) Contour map of the vorticity field at $t=3.5$ days. Contours (negative relative to the mean are dashed and positive solid) run from -100 to 90 at intervals of 10 non-dimensional units. (b) Spectra of kinetic energy variable $\mathcal{R}^{\prime}$ associated with the third (thin solid) and fifth (thin dashed) baroclinic modes after a simulation duration of 3.5 days. Also shown are the spectrum slopes deduced from the analytical solution using $n=1.7$ (thick solid).

the spectral peaks after 3.5 days are 12 and 22 for the third and fifth baroclinic modes respectively, whereas (7) leads to $k_{\mathrm{c}}$ values of 12 and 24 respectively. Furthermore, numerical values for these spectral peaks are $\left|\widehat{\mathcal{R}}_{k}^{\prime}\right|^{2}=7 \times 10^{-3}$ and $2 \times 10^{-3}$ for the third and fifth modes respectively, close to the estimates using (8), which are $8 \times 10^{-3}$ and $3 \times 10^{-3}$. For all spectra, the spectral peak (close to $k_{\mathrm{c}}$ ) separates two distinct regions (Fig. 1(b)): (i) a large-scale region with a positive spectral slope close to $k^{2.3}$, i.e. close to that of $\nabla^{2} Z$, and (ii) a small-scale region with a negative spectral slope close to $k^{-5.7}$, i.e. close to that of the stream function. These slopes almost match the predictions of (8) with $n=1.7$ (see the thick solid lines on Fig. 1(b)). A detailed examination of the numerical results indicates that the amplitude of the spectra in the large-scale region (for $k$ smaller than the peak) grows with time (as expected from (8)), whereas it is steady in the small-scale region (for $k$ larger than the peak). Hence the spectral peak moves to smaller $k$. This behaviour confirms the analytical analysis.

In physical space, we found quite good agreement during the first few days between numerical and analytical solutions for $\mathcal{R}$. Figures 2(a) and 3(a) show the horizontal distribution of $\mathcal{R}^{\prime}$, for the third and fifth baroclinic modes at $t=3.5$ days. The corresponding analytical solutions (cf. (9)) are shown on Figs. 2(b) and 3(b). They correspond to the truncated Laplacian vorticity field (i.e. $\nabla^{2} Z$, filtered to retain only Fourier modes with $k<k_{\mathrm{c}}\left(k_{\mathrm{c}}=12\right.$ for the third mode and 24 for the fifth mode at this time), scaled with $h t^{2} / 8$. The spatial structures of the numerical and analytical solutions match well both in location and magnitude. They are characterized by small-scale elongated structures with both positive and negative values. Their amplitudes can reach values from -1 up to 2.25 for the third mode and -0.75 to 2.5 for the fifth mode. Regions with high $\mathcal{R}^{\prime}$-amplitude are located in the same areas as those where $\nabla^{2} Z_{T}$ has a large amplitude. This resemblance is clear in Figs. 2(c) and 3(c), which show the values of the numerical and analytical solutions along the lines indicated on Figs. 2(a) and (b) and 3(a) and (b); both the location of the minima and maxima of $\mathcal{R}^{\prime}$ and their amplitudes are well 
(a)

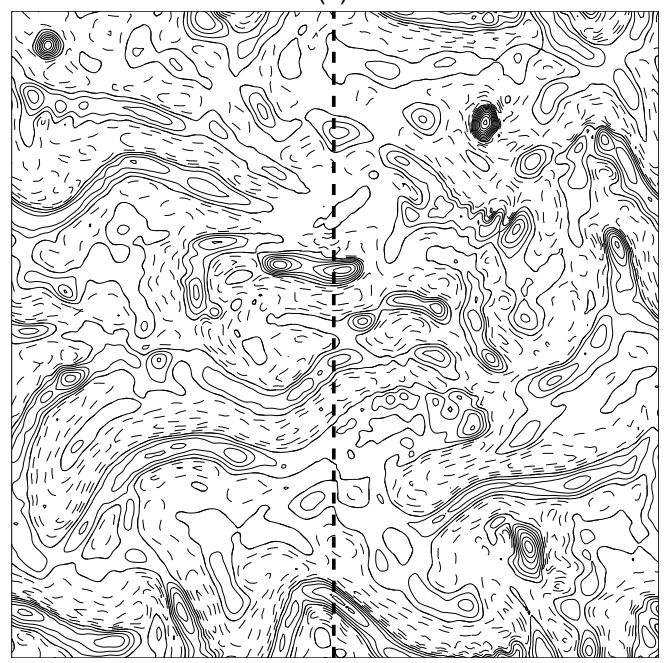

(b)

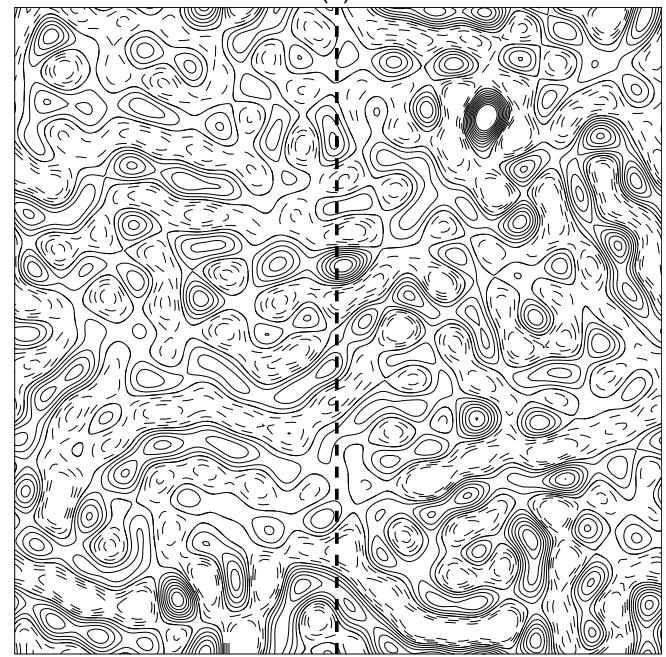

(c)

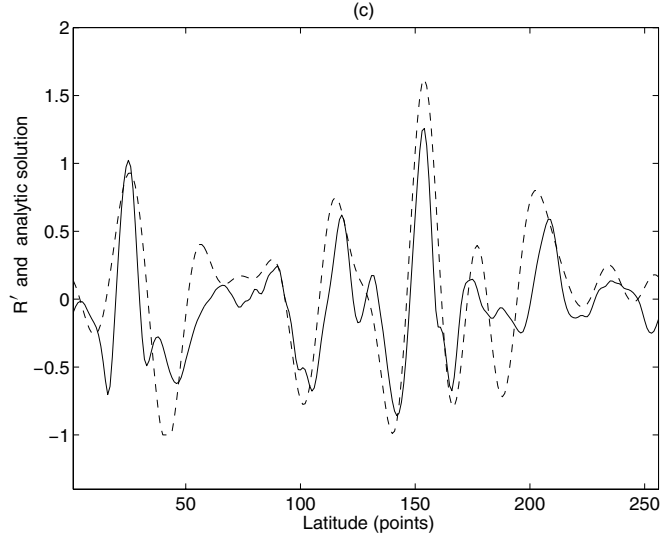

Figure 2. Contour maps at $t=3.5$ days of (a) kinetic energy variable $\mathcal{R}^{\prime}$ (third mode) and (b) the corresponding analytical solution (for which $k_{\mathrm{c}}=12$ ). Contours (negative relative to the mean are dashed and positive solid) run from -0.75 to 2.25 at intervals of 0.25 non-dimensional units. (c) shows the $\mathcal{R}^{\prime}$-values along the thick dashed lines shown on (a) and (b), for the third mode (solid) and the analytical solution (dashed).

reproduced by the analytical solutions. Comparison with the vorticity field (Fig. 1(a)) reveals that positive $\mathcal{R}^{\prime}$-structures are always located on the anticyclonic side of strong vorticity fronts. However the characteristic patterns of the third and fifth modes differ; the fifth mode involves smaller scale than the third one. These differences appear to be well captured by the analytical solution. There are however some disparities between the analytical and numerical solutions; the spatial patterns of the analytical solution are more scattered and patchy, and less elongated (Fig. 2(b)). Sensitivity tests (not shown) reveal that this is due to the absence of the effects of the advective terms in this solution which usually make these patterns more elongated and less scattered. A numerical simulation using (2) without the advective terms has confirmed this feature. However, despite these slight disparities, the location and amplitude of the NIO kinetic energy appears to be well reproduced by the analytical solution at this time. This strong resemblance indicates that the scales of the structures where NIO energy is trapped in the numerical solution are close to $k_{\mathrm{c}}^{-1}$ (which is the dominant scale of the analytical solution). 
(a)

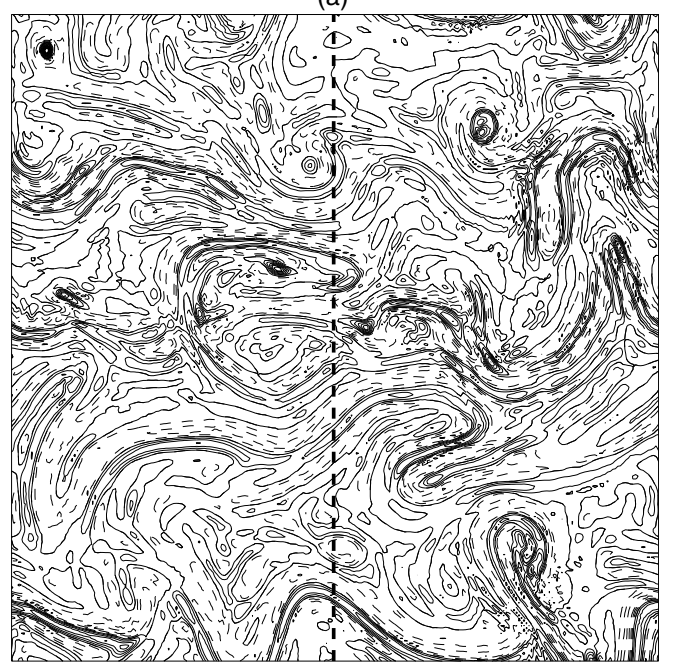

(b)

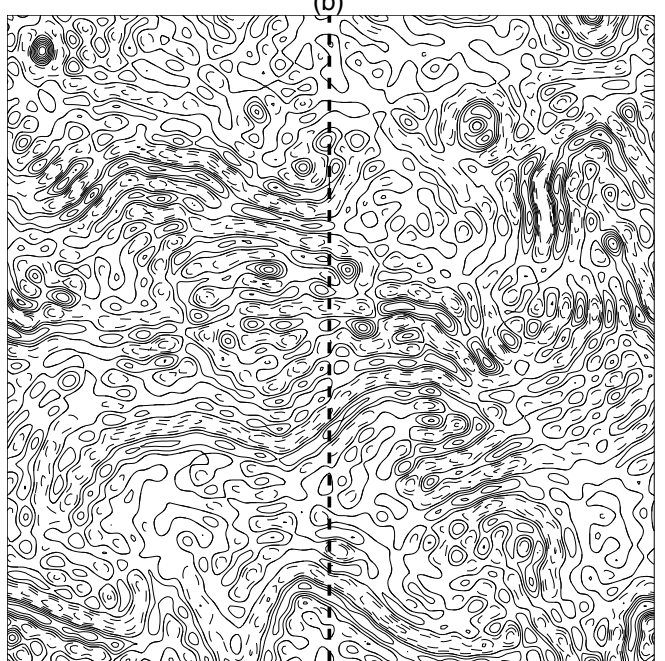

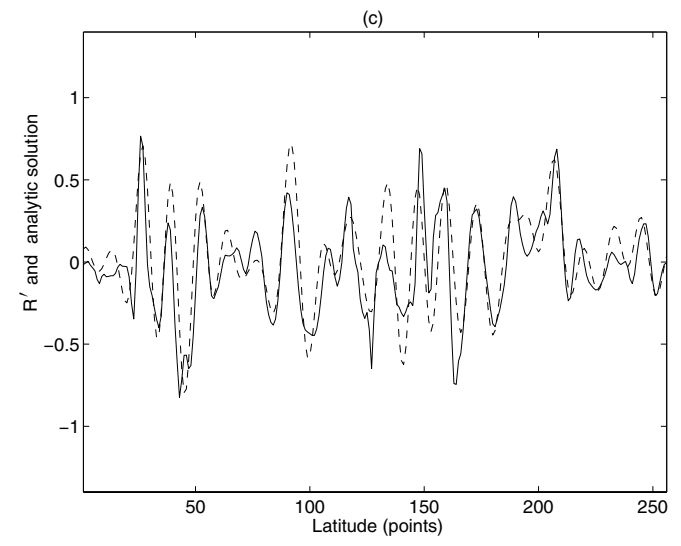

Figure 3. Same as Fig. 2 but for the fifth mode (with $k_{\mathrm{c}}=24$ in (b)).

The scatter plots displayed on Figs. 4(a) and (b) confirm the good correspondence at this time ( $t=3.5$ days) between the numerical and analytical solutions. In particular, the correspondence is very good for $\left|\mathcal{R}^{\prime}\right|<0.4$, which confirms that the assumptions leading to (4) work well in this range. There are however two differences: (i) the average amplitude of the minima appear to be underestimated by the analytical solution and (ii) the dispersion is more significant near the extrema. We have no clear explanation for the underestimated minima, but the dispersion near the extrema is explained by a small spatial shift in the location of the two solutions, as revealed by Figs. 2(c) and 3(c). In addition, we have checked the sensitivity of the resemblance between the numerical and analytical solutions to the choice of $k_{\mathrm{c}}$. When using a $\nabla^{2} Z_{T}$ field truncated at $k_{\mathrm{c}}=10$ (or 16) instead of $k_{\mathrm{c}}=12$ for the third mode, the location of the extrema is less well reproduced and their amplitude is underestimated (overestimated) by more than $20 \%$. For the higher baroclinic modes (for which $k_{\mathrm{c}}$ is larger), the correspondence between the numerical and analytical solutions is much more sensitive to the choice of the value of $k_{\mathrm{c}}$. The reason is that, because of the positive Laplacian vorticity spectrum slope $\left(k^{2.3}\right)$, the contribution of the spectral band close to $k_{\mathrm{c}}$ is larger as $k_{\mathrm{c}}$ is larger. 

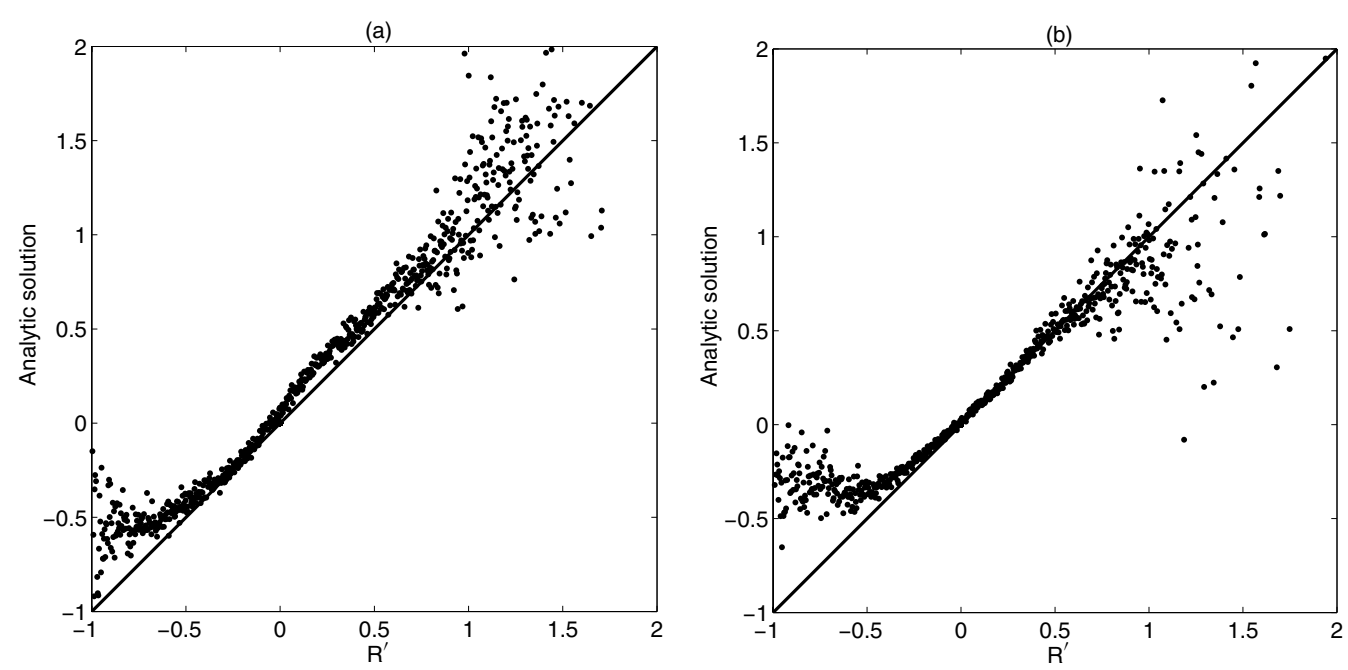

Figure 4. Scatter plots of kinetic energy variable $\mathcal{R}^{\prime}$ and the corresponding analytical solution for the (a) third and the (b) fifth modes at $t=3.5$ days. The dots represent averages over grid points for $\mathcal{R}^{\prime}$ within \pm 0.001 of the central value given in the abscissa.

However, for larger times ( $t=7$ days), the resemblance between the numerical and analytical solutions is not so compelling. The location and amplitude of the extrema of the NIO energy are not so well reproduced by the analytical solution. This means that nonlinear triad interactions (included in the advection terms and the term involving the vorticity), neglected in the analytical solution, become important.

Thus, despite some disparities, the comparison between the numerical and analytical solutions confirms the robustness of the analytical analysis. It is the competition between vorticity and dispersion effects that leads to the rapid spatial organization of the inertial energy; on a short time-scale (a few days) compared to the eddy advective time-scale (30 days), inertial energy is concentrated in structures with positive $\nabla^{2} Z$ with a size close to $k_{\mathrm{c}}^{-1}$, and the amplitude of the inertial energy is proportional to $\nabla^{2} Z$ in those structures. These areas are mostly located on the anticyclonic side of strong vorticity fronts.

\section{NUMERICAL ASSESSMENT USING A SHALLOW-WATER MODEL}

One may wonder whether the assumptions of YBJ97 may limit the validity of our results. To address this point, we have compared the preceding results with those from a shallow-water model (SWM). This model may be viewed as a reduction of the primitive equations to two layers, one active and the other at rest. The governing equations are:

$$
\left.\begin{array}{c}
\frac{\mathrm{d}_{\mathrm{H}}}{\mathrm{d} t} u+u \frac{\partial u}{\partial x}+v \frac{\partial u}{\partial y}+u \frac{\partial U_{\mathrm{g}}}{\partial x}+v \frac{\partial U_{\mathrm{g}}}{\partial y}-f v=-g^{\prime} \frac{\partial h}{\partial x}+v \nabla^{2} u \\
\frac{\mathrm{d}_{\mathrm{H}}}{\mathrm{d} t} v+u \frac{\partial v}{\partial x}+v \frac{\partial v}{\partial y}+u \frac{\partial V_{\mathrm{g}}}{\partial x}+v \frac{\partial V_{\mathrm{g}}}{\partial y}+f u=-g^{\prime} \frac{\partial h}{\partial y}+v \nabla^{2} v \\
\frac{\mathrm{d}_{\mathrm{H}}}{\mathrm{d} t} h+\frac{\partial u h}{\partial x}+\frac{\partial v h}{\partial y}=0,
\end{array}\right\}
$$

where $\mathrm{d}_{\mathrm{H}} \cdot \mathrm{d} t=\partial \cdot / \partial t+U_{\mathrm{g}} \partial \cdot / \partial x+V_{\mathrm{g}} \partial \cdot / \partial y$ and $v$ is a horizontal diffusion coefficient. $u$ and $v$ are the velocities associated with the inertial oscillations, and $U_{\mathrm{g}}$ and $V_{\mathrm{g}}$ 
(a)

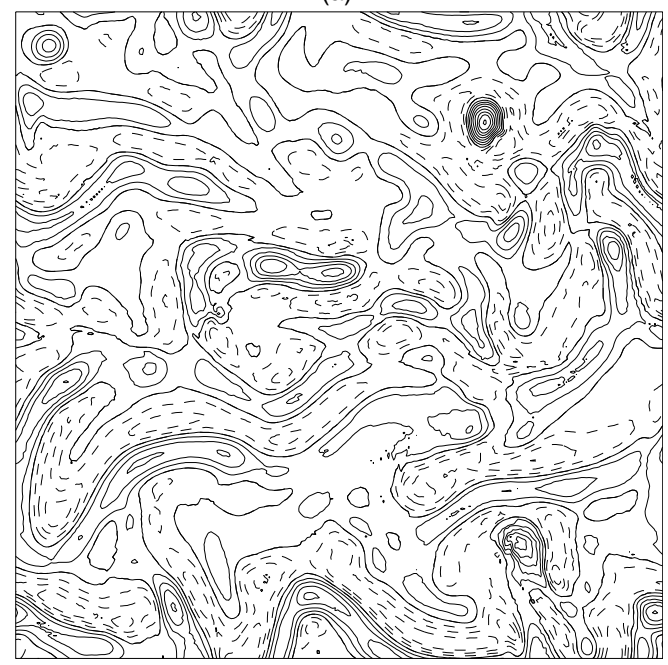

(b)

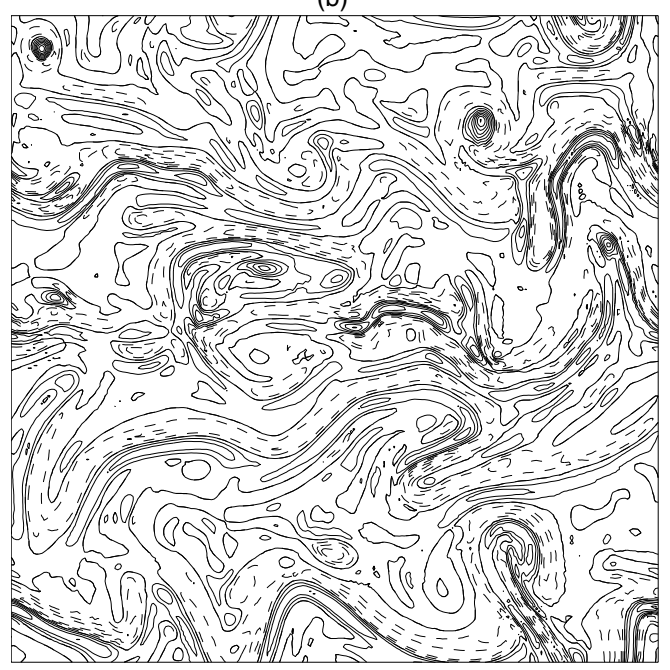

Figure 5. Contour maps at $t=3.5$ days of kinetic energy variable $\mathcal{R}^{\prime}$ for the (a) third and (b) fifth modes from the shallow-water model. Contours (negative relative to the mean are dashed and positive solid) run from -0.75 to 2.25 at intervals of 0.25 non-dimensional units.

those associated with the mesoscale eddy field used in the preceding section. This model hence provides a decomposition of the dynamics into a background QG flow and an evolving NIO field, with no feedback from the latter onto the former. $h$ designates in this section the mixed-layer depth. Its initial value, $h_{0}$, and the reduced gravity, $g^{\prime}$, have been chosen to correspond to the Rossby radius of deformation $\left(r_{\mathrm{d}}=\sqrt{g^{\prime} h_{0}} / f\right.$, with $f=0.7 \times 10^{-4} \mathrm{~s}^{-1}$ ) of the third and fifth NIO modes. New nonlinear terms, which are neglected in the YBJ97 procedure, are included in (10), i.e. those related to the inertial wave interactions (the second and third terms) and to the eddy strain effects (included in the fourth and fifth terms). Numerical simulations have been performed with the SWM. The NIO energy at $t=0$ is again uniform with a value $h\left(u^{2}+v^{2}\right) / 2=1 \mathrm{~m}^{3} \mathrm{~s}^{-2}$. The numerical resolution is $512 \times 512$. For a better comparison with the results using the YBJ97 equations, the kinetic energy associated with the NIOs has been averaged over one inertial period in order to recover $\mathcal{R}$, the subinertial amplitude of the inertial motions.

Figures 5(a) and (b) show the results in physical space for the third and fifth NIO modes and can be compared directly with Figs. 2(a) and 3(a). The spatial structures of the two $\mathcal{R}^{\prime}$-fields match each other. The locations of their extrema appear to be the same and their scales are comparable. However, the small scales appear to be slightly less energetic in the SWM solutions. Comparison of Figs. 5(a) and (b) with Figs. 2(b) and 3(b) reveals that the analytical solution (9) still captures the spatial structure of the $\mathcal{R}^{\prime}$-field for the third and fifth baroclinic modes; the location of the extrema as well as their amplitudes are well reproduced. We have performed additional experiments without the second and third terms in (10) and with $h$ replaced by $h_{0}$ in (11). The results (not shown) during the first 4 days are very close to those using the full SWM. Hence the nonlinear terms involving near-inertial wave interactions have almost no influence on the evolution of the NIO field. On the other hand, the advection terms involving the QG flow have been found to have a non-negligible effect; as found from the YBJ97 solution, they lead to the spatial heterogeneity of the NIO energy being less scattered 
than it is with the analytical solution. All these sensitivity tests confirm the robustness of the YBJ97 procedure, and that it is principally the competition between vorticity and dispersion effects that determines the characteristics of the spatial organization of the inertial energy on a short time-scale. This explains why the analytical solution works well at these short time-scales.

\section{DisCUSSION}

The results of the present study show that, on a time-scale of a few days, the Laplacian of the vorticity field is the main factor that drives the spatial organisation of NIO kinetic energy and its time evolution. Furthermore, there is a critical timedependent wave number above which NIO energy is small. This indicates that NIO energy is well mixed over a length-scale corresponding to this wave number. Thus only the Laplacian of the vorticity, truncated at this scale, is relevant in characterizing the spatial structure of NIO energy.

A heuristic explanation can be advanced. As shown by previous studies, because of the vorticity effects, phase differences accumulate in NIOs within mesoscale structures, leading to spatial variability of these oscillations close to the vorticity field. Then, because of dispersion effects that involve the second spatial derivatives, spatial variability in the NIO amplitude (or kinetic energy), that is for very early times close to the Laplacian of the vorticity, builds up. If the eddy field is characterized by only one energetic length-scale, i.e. if the vorticity spectrum has a peak corresponding to this scale and a slope steeper than $k^{-4}$, then the Laplacian vorticity spectrum slope is negative and the dispersion effects, like the vorticity effects, are mostly energetic at this lengthscale. In this case, the spatial distribution of the NIO energy should be characterized by only this energetic length-scale and should slowly evolve towards the stream function field (as suggested by YBJ97). However, when the vorticity spectrum slope is shallower than $k^{-4}$, the mechanisms are quite different. In this case, vorticity effects are still mostly efficient at large scales, but the Laplacian vorticity spectrum slope is positive and therefore the dispersion effects are the most efficient at the smallest scale. This scale is actually determined by $k_{\mathrm{c}}$ and, as discussed by Metzger (1999), it is the scale over which NIO energy is mixed. The arguments proposed by Metzger (1999) involve $f r_{\mathrm{d}}^{2}$, named a 'dispersivity' parameter analogous to the diffusivity associated with passive scalar diffusion processes. Using this dispersivity parameter, the length-scale, $\delta$, over which the NIOs are dispersed is given by $\delta=r_{\mathrm{d}} \sqrt{\pi f t}$.

Another aspect of our results is the quite rapid and significant evolution of the organisation of the NIO kinetic energy induced by the eddy field. The length-scale of the eddies is $\mathcal{O}(200 \mathrm{~km})$ and their advective time-scale is $\mathcal{O}(30$ days $)$. After just one tenth of this time-scale, the inertial energy, which is initially homogeneous, displays spatial variation on space-scales of the order of one tenth of the eddy spatial scale with energy fluctuations between one third and twice the initial amplitude. For larger times (i.e. after 7 days), the analytical solution does not work. For these times, numerical results display a saturation due to the nonlinear advection terms which lead to the NIO energies being scattered within the mesoscale structures. However we believe that our results, and in particular the proposed analytical solution, still apply to the real ocean. Indeed, the situation envisaged is the response of the upper oceanic layers after a wind event, and the time interval between two wind events is usually of the order of some days (Alford 2001).

What can we deduce from our results for the vertical propagation of the NIOs? This propagation can be interpreted in terms of the different behaviour of their baroclinic 
modes (Gill 1984). The direct consequence of a storm is the generation of strong NIOs within the mixed layer. In terms of baroclinic modes, the amplitude and the phase of the modes are such that these modes balance each other below the mixed layer, yielding zero velocity there. Because of the effects of the eddies, each mode has a phase and an amplitude that evolve differently from the others. Consequently, a phase shift between the modes builds up with time and their relative amplitudes become different. This leads to an imbalance between the modes and therefore to a decrease of the velocity within the mixed layer and the appearance of a non-zero velocity below the mixed layer (Wang 1991; D'Asaro et al. 1995; Klein and Treguier 1995; Balmforth et al. 1998; Van Meurs 1998). Within this context, our results yield more precise information on the vertical propagation of the inertial energy. The spatial variability of the phase shift grows with time and is proportional to the vorticity field (see Kunze 1985 and (3)). Therefore its dominant length-scale is close to that of the eddies. The spatial variability of the amplitude also builds up with time (due to the additional effects of dispersion) but, as revealed by this study, it involves length-scales that are much smaller than that of the vorticity field. Indeed, after 3.5 days, the dominant length-scales of the NIO kinetic energy are 3 times and 6 times (for the third and fifth modes respectively) smaller than the dominant scale of the vorticity field. The outcome is that the vertical propagation of NIOs should involve mostly small horizontal scales. One consequence of the present results is that the response of the upper ocean to a series of successive wind impulses with a time period of some days may reinforce the physics described in this study. This may strongly enhance the effects of time variability of the wind stress on the mixing of the upper ocean. A future study should investigate these effects.

Finally, we have assumed that the mesoscale eddy field is homogeneous over the water column. This stems from the fact that usually the vertical scale of the mesoscale eddies is much larger than the characteristic vertical scale of the NIOs. This assumption allows us to interpret the vertical propagation of the NIO energy in terms of mode separation using the vertical normal-mode approach of Gill (1984). However, results may differ when the vertical scales of the eddy field are close to those of the NIOs. In that case, the possible effects of critical layers cannot be ignored and require an approach different from this study.

\section{CONCLUSIONS}

This study has investigated analytically and numerically the spatial reorganization of the kinetic energy of the NIOs by a turbulent eddy field, i.e. a field involving a large number of strongly interacting eddies characterized by a small Rossby number. The main results are:

- Within just a few days, the eddies efficiently reorganize an initially uniform NIO kinetic energy field such that the spatial heterogeneity of the inertial energy resembles not the vorticity or the stream function, as previous studies would suggest, but the Laplacian of the vorticity. The properties of this reorganization are well described by an analytical solution. This is the new result of this study.

- This solution, confirmed by numerical simulations using different models, requires only the knowledge of the Laplacian of the eddy vorticity (which can be deduced directly from the stream function) and the Rossby radius of deformation of the NIO mode considered.

- The corresponding structures where the inertial energy is trapped do not have scales smaller than a critical length-scale that increases with time and is proportional to the Rossby radius of deformation of the NIOs. 
- The analytical analysis has allowed us to provide a physical explanation for this solution. The physics rely on the assumption that the vorticity spectrum is shallower than $k^{-4}$. While the large-scale eddy vorticity structures affect the phase of the NIOs, as found by previous studies, dispersion effects that involve the second spatial derivatives and the shallowness of the vorticity spectrum slope explain why the inertial energy field resembles the Laplacian vorticity and why it has a peak at the critical length-scale mentioned previously.

- These results are only valid on time-scales of a few days, which is also the timescales between two wind events.

\section{ACKNOWLEDGEMENTS}

This work was supported by Institut Français de Recherche pour l'Exploitation des Mers (IFREMER), the Centre Nationale de la Recherche Scientifique and the Visiting Program of the Office of Naval Research International Field Office. Some of the calculations reported here were done on the NEC SX5 of the Institut du Développement et des Ressources en Informatique Scientifique, Orsay, France. Conversations with Bill Young were very helpful. We thank the editor and both referees whose comments led to an improved presentation of our results.

Alford, M. H.

Balmforth, N. J.,

Llewellyn Smith, S. G. and Young, W. R.

D’Asaro, E. A., Eriksen, C. C., Levine, M. D., Niiler, P.,

Paulson, C. A. and

Van Meurs, P.

Gill, A. E.

Hua, B. L. and Haidvogel, D. B.

Hurlburt, H. E. and Hogan P. J.

Klein, P. and Llewellyn Smith, S. G. 2001

Klein, P. and Treguier, A. M.

1995

Kunze, E.

1985

Metzger, M. M.

1999

Rudnick, D. L.

2001

Siegel, A., Weiss, J. B., Toomre, J., McWilliams, J. C.,

Berloff, P. S. and Yavneh, I.

Stammer, D.

2001

1997

Van Meurs, P.

1998

\section{REFERENCES}

2001

1998

1995

1984

1986

2000
Internal swell generation: the spatial distribution of energy flux from the wind to mixed-layer near-inertial motions. J. Phys. Oceanogr., 31, 2359-2368

Enhanced dispersion of near-inertial waves in an idealized geostrophic flow. J. Mar. Res., 56, 1-40

Upper-ocean inertial currents forced by a strong storm. Part I: Data and comparisons with linear theory. J. Phys. Oceanogr., 25, 2909-2936

On the behavior of internal waves in the wakes of storms. J. Phys. Oceanogr., 14, 1129-1151

Numerical simulations of the vertical structure of quasigeostrophic turbulence. J. Atmos. Sci., 43, 2923-2936

Impact of $1 / 8^{\circ}$ to $1 / 64^{\circ}$ resolution on Gulf Stream modeldata comparisons in basin-scale subtropical Atlantic Ocean models. Dyn. Atmos. Oceans, 32, 283-329

Horizontal dispersion of near-inertial oscillations in a turbulent mesoscale eddy field. J. Mar. Res., 59, 697-723

Dispersion of wind-induced inertial waves by a barotropic jet. J. Mar. Res., 53, 1-22

Near-inertial wave propagation in geostrophic shear. J. Phys. Oceanogr., 15, 544-565

'Scalar dispersion in a two-dimensional random flow field'. In Stirring and Mixing, 1999 Summer Study Program in Geophysical Fluid Dynamics, Woods Hole Oceanographic Institution.

On the skewness of vorticity in the upper ocean. Geophys. Res. Letters, 28, 2045-2048

Eddies and vortices in ocean basin dynamics. Geophys. Res. Letters, 28, 3183-3186.

Global characteristics of ocean variability estimated from regional TOPEX/POSEIDON altimeter measurements. J. Phys. Oceanogr., 27, 1743-1769

Interactions between near-inertial mixed layer currents and the mesoscale: the importance of spatial variabilities in the vorticity field. J. Phys. Oceanogr., 28, 1363-1388 
Wang, D. P.

Weller, R. A.

Wunsch, C.

Young, W. R. and Ben Jelloul, M.
1991 Generation and propagation of inertial waves in the subtropical front. J. Mar. Res., 49, 619-633

1982 The relation of near-inertial motions observed in the mixed layer during the JASIN (1978) experiment to the local wind stress and to the quasi-geostrophic flow field. J. Phys. Oceanogr., 12, 1122-1136

Oceanogr., 27, 1770-1794

1997 Propagation of near-inertial oscillations through a geostrophic flow. J. Mar. Res., 55, 735-766 